\title{
Tongue squamous cell carcinoma as a possible distinct entity in patients under $\mathbf{4 0}$ years old
}

\author{
QI-GEN FANG ${ }^{1}$, SHUANG SHI $^{2}$, FA-YU LIU ${ }^{1}$ and CHANG-FU SUN ${ }^{1}$ \\ Departments of ${ }^{1}$ Oral and Maxillofacial Surgery and ${ }^{2}$ Pediatric Dentistry, School of Stomatology, \\ China Medical University, Shenyang, Liaoning 110002, P.R. China
}

Received September 18, 2013; Accepted March 14, 2014

DOI: $10.3892 / \mathrm{ol} .2014 .2054$

\begin{abstract}
Much controversy exists in the published literature regarding the clinical course and prognosis of tongue squamous cell carcinoma (SCC) in young patients. The aim of the current study was to evaluate the clinical results of tongue SCC in young patients. A total of 176 patients were included in this retrospective study. The patients were divided into two groups (young and old) according to an age cut-off of 40 years. The $\chi^{2}$ test and Kaplan-Meier method were used to analyze the variables. In total, 15 patients were $<40$ years old and placed into the young group, with five-year recurrence-free survival (RFS) and disease-specific survival (DSS) rates of 30 and $63 \%$, respectively, compared with 47 and $62 \%$, respectively, in the old group. No significant differences were identified between the RFS and DSS rates of the two groups, however, the young patients exhibited a different failure pattern. Overall, nine out of 10 recurrences in the young group occurred at a primary site compared with 18 out of 70 in the old group $(\mathrm{P}<0.001)$. Univariate analysis revealed that gender and differentiation were associated with recurrence and neck nodal involvement. In addition, poor differentiation was found to significantly decrease the DSS time. However, the prognosis of tongue SCC in the young patient group did not appear to differ from that of the old patient group. Furthermore, in the young patient group, local recurrence was the most common failure pattern and tumor differentiation was the most important prognostic factor.
\end{abstract}

\section{Introduction}

Tongue squamous cell carcinoma (SCC) is the most common type of malignant tumor of the oral cavity, and usually occurs following the fifth decade of life. A previous study

Correspondence to: Professor Chang-Fu Sun, Department of Oral and Maxillofacial Surgery, School of Stomatology, China Medical University, 117 Nanjing North Street, Shenyang, Liaoning 110002, P.R. China

E-mail: qigenfang@hotmail.com

Key words: oral cancer, squamous cell carcinoma, young patients, oral tongue reported that $<3 \%$ of these carcinomas develop in patients of $<40$ years old (1). In this subgroup of patients, the proportion of females was greater and a history of smoking and drinking was less frequent. A great deal of controversy exists regarding the clinical course and prognosis of tongue SCC between young and old patients in the published literature. Certain studies have reported that young patients exhibit improved clinical results when compared with old patients $(2,3)$, whereas other studies have reported a significant decrease in the survival rate of young patient groups (4-6). However, further studies have revealed a similar prognosis between young and old patient groups (7-16).

In the present study, young and old patients with tongue SCC were compared to analyze disease recurrence and survival rates.

\section{Materials and methods}

Patient presentation. Approval for the current study was obtained from the Institutional Research Committee of the China Medical University (Shenyang, China). Between 2005 and 2011, 216 patients were treated for tongue SCC at the Oral Maxillofacial Head and Neck Tumor Center of China Medical University (Shenyang, China), however, 40 of these patients were excluded from the present study, as they were lost to follow-up. Therefore, a total of 176 patient medical records were reviewed. The patient information, including demographic data, tobacco and alcohol consumption, tumor stage, node stage, differentiation, recurrence and survival, were collected, and the patients were divided into two groups (young and old) according to an age cut-off of 40 years. Written informed consent was obtained from all patients.

Statistical analysis. The $\chi^{2}$ test was used to evaluate the significance of the variables and the Kaplan-Meier method was used to analyze the recurrence-free survival (RFS) and disease-specific survival (DSS) rates. Statistical analysis was conducted using SPSS version 13.0 (SPSS, Inc., Chicago, IL, USA) and $\mathrm{P}<0.05$ was considered to indicate a statistically significant difference.

\section{Results}

Patient characteristics of the young group. The young group consisted of 15 patients (six male and nine female), with an average age of 34.0 years (range, $22-40$ years). In total, $33.3 \%$ of the patients had a history of tobacco use and $13.3 \%$ of patients 
Table I. Summary of patient information.

\begin{tabular}{|c|c|c|c|}
\hline Parameters & Young group $(n=15)$ & Old group $(n=161)$ & P-value ${ }^{a}$ \\
\hline Age, years (range) & $34.0(22-40)$ & $57.9(42-94)$ & $<0.001$ \\
\hline \multicolumn{4}{|l|}{ Gender $[\mathrm{n},(\%)]$} \\
\hline Male & $6(40.0)$ & $113(70.2)$ & \multirow[t]{2}{*}{0.023} \\
\hline Female & $9(60.0)$ & $48(29.8)$ & \\
\hline \multicolumn{4}{|l|}{ Habits $[\mathrm{n},(\%)]$} \\
\hline Tobacco use & $5(33.3)$ & 109 (67.7) & 0.008 \\
\hline Alcohol consumption & $2(13.3)$ & $72(44.7)$ & 0.026 \\
\hline \multicolumn{4}{|l|}{ Tumor stage $[\mathrm{n},(\%)]$} \\
\hline $\mathrm{T} 1$ & $4(26.7)$ & $25(15.5)$ & \multirow[t]{4}{*}{0.484} \\
\hline $\mathrm{T} 2$ & $8(53.3)$ & $87(54.1)$ & \\
\hline $\mathrm{T} 3$ & $0(0.0)$ & $16(9.9)$ & \\
\hline $\mathrm{T} 4$ & $3(20.0)$ & $33(20.5)$ & \\
\hline \multicolumn{4}{|l|}{ Node stage $^{\mathrm{b}}[\mathrm{n},(\%)]$} \\
\hline N0 & $14(93.3)$ & $121(75.2)$ & \multirow[t]{3}{*}{0.329} \\
\hline N1 & $1(6.7)$ & $26(16.1)$ & \\
\hline $\mathrm{N} 2$ & $0(0.0)$ & $13(8.1)$ & \\
\hline \multicolumn{4}{|l|}{ Differentiation $[\mathrm{n},(\%)]$} \\
\hline Well & $6(40.0)$ & $85(52.8)$ & \multirow[t]{3}{*}{0.677} \\
\hline Moderate & $4(26.7)$ & $36(22.4)$ & \\
\hline Poor & $5(33.3)$ & $40(24.8)$ & \\
\hline \multicolumn{4}{|l|}{ Treatment $[\mathrm{n},(\%)]$} \\
\hline Surgery & $10(66.7)$ & $99(61.5)$ & \multirow[t]{3}{*}{0.648} \\
\hline Surgery + radiation & $5(33.3)$ & $50(31.1)$ & \\
\hline Surgery + radiation + chemotherapy & $0(0.0)$ & $12(7.5)$ & \\
\hline Follow-up, months (range) & $38.7(8-96)$ & $37.9(6-96)$ & 0.566 \\
\hline
\end{tabular}

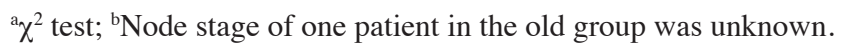

had a history of alcohol consumption. Of the 15 tumors, four (26.7\%) were staged as T1, eight (53.3\%) as T2 and three (20\%) as T4. Furthermore, six tumors (40\%) were well-differentiated, four tumors $(26.7 \%)$ were moderately-differentiated and five tumors $(33.3 \%)$ were poorly-differentiated. The mean follow-up time was 38.7 months (range, 8-96 months) (Table I).

Patient characteristics of the old group. The old patient group consisted of 161 patients (113 male and 48 female), with an average age of 57.9 years (range, 42-94 years). In total, $67.7 \%$ of the patients had a history of tobacco use and $44.7 \%$ had a history of alcohol consumption. Of the 161 tumors, $25(15.5 \%)$ were staged as T1, $87(54.0 \%)$ as T2, $16(9.9 \%)$ as T3 and $33(20.5 \%)$ as T4. Furthermore, 85 tumors (52.8\%)were well-differentiated, 36 (22.3\%) were moderately-differentiated and $40(24.8 \%)$ were poorly-differentiated. The mean follow-up time was 37.9 months (range, 6-96 months) (Table I).

RFS rates. Fig. 1 compares the RFS rates of the two groups. In the young group 10 patients $(66.7 \%)$ exhibited locoregional recurrences (nine locally and one regionally) and the mean survival time until the first recurrence of the disease was 23.4 months (range, 6-72 months). In the old group, 58 patients
(36.0\%) exhibited locoregional recurrences (18 locally and 40 regionally) and 12 patients exhibited distant metastasis. Furthermore, the mean survival time until the first recurrence of the disease was 17.0 months (range, 3-96 months).

DSS rates. Fig. 2 compares the DSS rates of the two groups. In the young group, five patients succumbed to the disease and the mean survival time prior to mortality was 16.8 months (range, 8-27 months). In the old group, 44 patients $(27.3 \%)$ succumbed to the disease and the mean survival time prior to mortality was 20 months (range, 7-65 months).

Univariate analysis. Univariate analysis of the young group suggested correlation between RFS and gender $(\mathrm{P}=0.059)$ and significant correlation with tumor differentiation $(\mathrm{P}=0.016)$, whereas node metastasis $(\mathrm{P}=0.016)$ and tumor differentiation $(\mathrm{P}=0.041)$ were found to significantly correlate with DSS.

\section{Discussion}

Traditionally, tongue SCC arises in middle-aged male patients following decades of tobacco and alcohol abuse, however, more recent studies have reported the increased incidence 


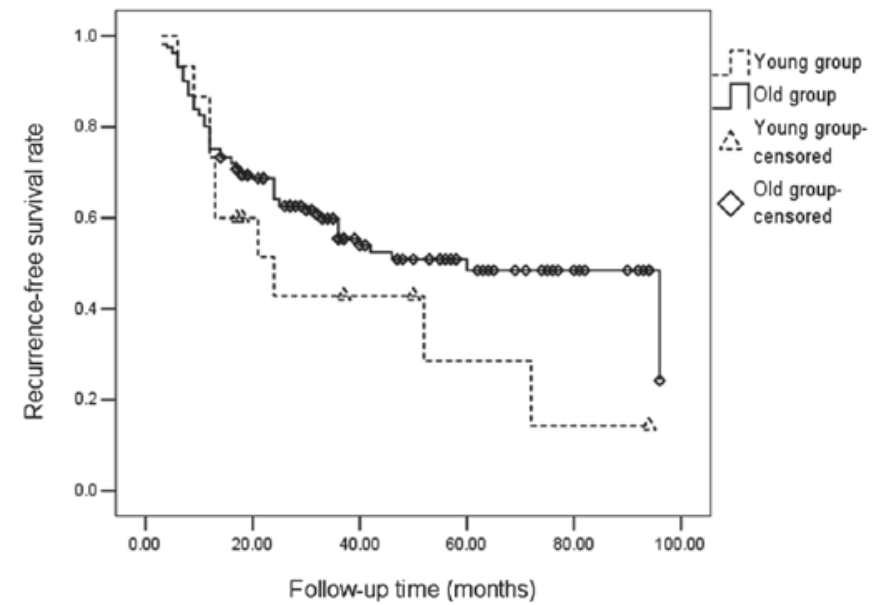

Figure 1. Comparison of recurrence-free survival (RFS) rates between the young and old patient groups $(\mathrm{P}=0.153)$.

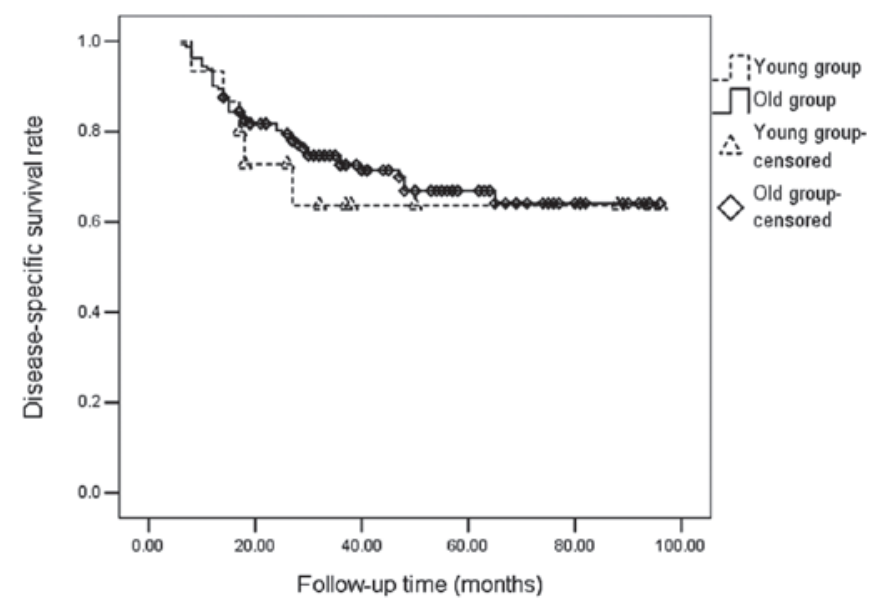

Figure 2. Comparison of disease-specific survival (DSS) rates between the young and old patient groups $(\mathrm{P}=0.631)$.

of tongue SCC in young individuals. Park et al (5) reported that $23(27.1 \%)$ out of 85 patients with tongue SCC were $<40$ years old, while Liao et al (11) reported that $76(25.8 \%)$ out of 296 patients with tongue SCC were $<40$ years old. However, the current study identified that the patients with tongue SCC who were $<40$ years old only presented $8.5 \%$ of the total patients.

Similar to the studies by Popovtzer et al (10), Friedlander et al (7) and Yip et al (14), the present study identified a higher percentage of females, non-smokers and non-drinkers in the young group compared with the old group. In addition, univariate analysis revealed that the female gender tended to be predictive of an improved RFS rate $(\mathrm{P}=0.059)$. However, Vargas et al (6) reported that young females with $\mathrm{SCC}$ of the anterior tongue exhibited significantly higher rates of recurrent disease.

Furthermore, consistent with the study by Pitman et al (13), the present study identified that the tumors of the young group appeared to be weakly aggressive at diagnosis; the majority of tumors (80\%) were staged as T1-T2 and only one tumor exhibited positive node metastasis. However, the clinical result was poor, as $10(66.7 \%)$ patients exhibited recurrence and five (33\%) patients succumbed to the disease. In addition, the univariate analysis revealed that positive nodal metastasis was found to significantly correlate with a decreased DSS time $(\mathrm{P}=0.016)$. Manuel et al (9) also reported a similar observation in which the five-year DSS of patients with histologically-positive nodes was significantly shorter compared with that of patients with pathologically-negative nodes. Additionally, Myers et al (17) reported that node stage was found to significantly correlate with decreased survival.

In the current study, no difference was observed in the differentiation distribution between the two age groups $(\mathrm{P}=0.677)$, a result which has been confirmed by Park et al (5), but is contradicted by other studies. Veness et al (18) reported that the proportion of poorly-differentiated tumors in the young group was greater, however, Manuel et al (9) and Atula et al (15) reported that the majority of the tumors in their series were well-differentiated. In the present study, tumor differentiation was identified to be the most important prognostic factor for young patients and was found to significantly correlate with the RFS $(\mathrm{P}=0.016)$ and DSS $(\mathrm{P}=0.041)$ rates. Similarly, Siegelmann-Danieli et al (16) concluded that tumor pathogenesis tended to predict clinical course, and Atula et al (15) revealed that moderately- to poorly-differentiated carcinomas exhibited a poor prognosis.

No overall consensus concerning the difference in prognosis between young and old groups has been reached, however, more recent studies have indicated similar clinical results (3-10). Furthermore, the current study indicated that the prognosis of the young patients markedly resembled that of the old patients. However, a trend was identified for the young patients to exhibit an increased rate of locoregional recurrence $(66.7 \%$ vs. $36.0 \%)$ and a decreased rate of distant metastasis (0 vs. $7.5 \%$ ) compared with the old patients. Additionally, nine out of 10 recurrences in the young group occurred at a primary site compared with 18 out of 70 recurrences in the old group, and this difference was found to be significant $\left(\chi^{2}\right.$ test; $\left.P<0.001\right)$. Local failure was more common in the young patients, and the possible causes were hypothesized to be due to the majority of tumors $(60 \%)$ being moderately- or poorly-differentiated and the fact that only one tumor exhibited neck nodal involvement in the young patients. Pitman et al (13) and Garavello et al (4) found that local recurrence was more common in young patients, however, Pitman et al (13) also reported that regional failure and distant metastasis were similar among young patients and controls. Furthermore, Friedlander et al (7) revealed a trend toward increased primary and regional recurrence in the young population and Soudry et al (8) reported a higher percentage of distant failure in the young group compared with the old group.

Notably, the present study observed that failure at the primary site carried a poor prognosis in the young group. In total, five out of nine young patients with local recurrences succumbed to the disease and the majority of the mortalities $(80 \%)$ occurred within two years of the initial surgery. Popovtzer et al (10) also revealed that young patients exhibit an aggressive course, with $40 \%$ mortality during the first two years.

In conclusion, the RFS and DSS rates identified in the current study were similar between the young and old groups, however, the failure pattern appeared to vary. The young 
patients exhibited an increased chance of local recurrence, and univariate analysis revealed that tumor differentiation was the most important prognostic factor for the young patients.

\section{References}

1. Son YH and Kapp DS: Oral cavity and oropharyngeal cancer in a younger population. Review of literature and experience at Yale. Cancer 55: 441-444, 1985.

2. Davidson BJ, Root WA and Trock BJ: Age and survival from squamous cell carcinoma of the oral tongue. Head Neck 23: 273-279, 2001.

3. Lee CC, Ho HC, Chen HL, Hsiao SH, Hwang JH and Hung SK: Squamous cell carcinoma of the oral tongue in young patients: a matched-pair analysis. Acta Otolaryngol 127: 1214-1217, 2007.

4. Garavello W, Spreafico R and Gaini RM: Oral tongue cancer in young patients: a matched analysis. Oral Oncol 43: 894-897, 2007.

5. Park JO, Sun DI, Cho KJ, Joo YH, Yoo HJ and Kim MS: Clinical outcome of squamous cell carcinoma of the tongue in young patients: a stage-matched comparative analysis. Clin Exp Otorhinolaryngol 3: 161-165, 2010.

6. Vargas H, Pitman KT, Johnson JT and Galati LT: More aggressive behavior of squamous cell carcinoma of the anterior tongue in young women. Laryngoscope 110: 1623-1626, 2000.

7. Friedlander PL, Schantz SP, Shaha AR, Yu G and Shah JP: Squamous cell carcinoma of the tongue in young patients: a matched-pair analysis. Head Neck 20: 363-368, 1998.

8. Soudry E, Preis M, Hod R, et al: Squamous cell carcinoma of the oral tongue in patients younger than 30 years: clinicopathological features and outcome. Clin Otolaryngol 35: 307-312, 2010.
9. Manuel S, Raghavan SK, Pandey M and Sebastian P: Survival in patients under 45 years with squamous cell carcinoma of the oral tongue. Int J Oral Maxillofac Surg 32: 167-173, 2003.

10. Popovtzer A, Shpitzer T, Bahar G, Marshak G, Ulanovski D and Feinmesser R: Squamous cell carcinoma of the oral tongue in young patients. Laryngoscope 114: 915-917, 2004.

11. Liao CT, Wang HM, Hsieh LL, et al: Higher distant failure in young age tongue cancer patients. Oral Oncol 42: 718-725, 2006.

12. Hyam DM, Conway RC, Sathiyaseelan Y, et al: Tongue cancer: do patients younger than 40 do worse? Aust Dent J 48: 50-54, 2003.

13. Pitman KT, Johnson JT, Wagner RL and Myers EN: Cancer of the tongue in patients less than forty. Head Neck 22: 297-302, 2000.

14. Yip CS, Charn TC, Wee JT, Tan TW, Goh C, Tan HK and Fong KW: Outcomes of oral tongue cancer: does age matter? Ann Acad Med Singapore 39: 897, 2010.

15. Atula S, Grénman R, Laippala P and Syrjänen S: Cancer of the tongue in patients younger than 40 years. A distinct entity? Arch Otolaryngol Head Neck Surg 122: 1313-1319, 1996.

16. Siegelmann-Danieli N, Hanlon A, Ridge JA, Padmore R, Fein DA and Langer CJ: Oral tongue cancer in patients less than 45 years old: institutional experience and comparison with older patients. J Clin Oncol 16: 745-753, 1998.

17. Myers JN, Elkins T, Roberts D and Byers RM: Squamous cell carcinoma of the tongue in young adults: increasing incidence and factors that predict treatment outcomes. Otolaryngol Head Neck Surg 122: 44-51, 2000.

18. Veness MJ, Morgan GJ, Sathiyaseelan Y and Gebski V: Anterior tongue cancer: age is not a predictor of outcome and should not alter treatment. ANZ J Surg 73: 899-904, 2003. 